科 学 通 报

\title{
关于无穷维线性系统稳定性的新结果 *
}

姚鸠飞冯德兴

(中国科学院系统科学研究所, 北京 100080)

\section{关链调 $C_{0}$ 半群、指数稳定性、Fourier 变换}

设 $H$ 是 Hilbert 空间, 其上的内积和范数分别记作〈・, ・ $\rangle$ 与 \|\| . 设 $A$ 是 $H$ 上线性算 子, $\rho(A)$ 表示 $A$ 的豫解集, $R(\lambda ; A)$ 表示 $A$ 的豫解算子, $\mathbb{R}=(-\infty, \infty)$. 就 Hilbert 空 间上 $C_{0}$ 半群的指数稳定性而言, 我们有

定理 1 设 $\tau(t)$ 是 $H$ 上线性算子 $A$ 生成的 $C_{0}$ 半群, 则 $\tau(t)$ 是指数稳定的充要条件 是

i) $\{\lambda \mid \operatorname{Re} \lambda>0\} \subset \rho(A)$;

ii) $\lim _{\sigma \rightarrow 0^{+}} \operatorname{asup} \tau \in R(a+i \tau ; A) \|=0$.

容易给出例子, 上述结论的充分性在 Banach 空间中不成立, 文献 [1] 中例 4.2 就是这样的 反例.

Pritchard 和 Zabczyk 在文献[2]中提出了一个公开的问题: 对于 $H$ 上的 $C_{0}$ 半群 $\tau(t)$, 如果存在 $p, p \geqslant 1$, 使

$$
\int_{0}^{\infty}|\langle\tau(t) x, y\rangle|^{p} \mathrm{~d} t<+\infty, \forall x, y \in H,
$$

问 $T(t)$ 是否是指数稳定的? 文献 [3]对 $p=1$ 的情况给出了肯定的回答. 本文中, 我们证明 了上述问题对 $1<p<+\infty$, 答案也是肯定的. 这就是

定理 2 设 $T(t)$ 是 $H$ 上 $C_{0}$ 半群, 则 $T(t)$ 是指数稳定的充要条件是存在 $p, 1 \leqslant p<$ $+\infty$,使(1)式成立.

如果我们从豫解式的角度来考虑问题, 对 $C_{0}$ 半群生成元豫解式限制类似于(1)的条件， 我们可得到

定理 3 设 $T(t)$ 是 $H$ 上线性算子 $A$ 生成的 $C_{0}$ 半群, 则 $T(t)$ 是指数稳定的当且仅当

i) $\{\lambda \mid \operatorname{Re} \lambda>0\} \subset \rho(A)$;

ii) 存在 $p, 1<p<+\infty$, 使得 $\forall x, y \in H$,

$$
\int_{\mathbf{R}}|\langle R(a+i \tau ; A) x, y\rangle|^{p} \mathrm{~d} z \leqslant c(x, y), \forall \sigma>0,
$$

其中 $c(x, y)$ 是与 $\sigma>0$ 无关的常数.

下面对上列三个定理分别给出证明.

定理 1 的证明 必要性. i) 是显然的, 只证 ii). 由文献 [3], 存在常数 $k>0$, 使

1992-11-06 收稿.

- 国家自然科学基金资助项目. 


$$
\|R(\lambda ; A)\| \leqslant k, \quad \forall \operatorname{Re} \lambda \geqslant 0,
$$

从而

$$
0 \leqslant \lim _{\sigma \rightarrow 0^{+}} \sigma \sup _{\tau \in \mathbb{R}}\|R(\lambda ; A)\| \leqslant \lim _{\sigma \rightarrow \sigma^{+}} \sigma k=0 .
$$

充分性.由 $\mathrm{ii}$ ),可取 $a_{0}>0$,使

$$
k_{0}=\sigma_{0} \sup _{\tau \in \mathbb{R}}\left\|R\left(\sigma_{0}+i \tau ; A\right)\right\|<1 \text {, }
$$

令

$$
c(\tau)=R\left(\sigma_{0}+i \tau ; A\right)\left[1-\sigma_{0} R\left(\sigma_{0}+i \tau ; A\right)\right]^{-1}, \forall \tau \in \mathbf{R},
$$

其中 $I$ 为 $H$ 上恒等算子. 由(3)式知 $c(\tau)$ 是 $H$ 上有界线性算子, 易知

$$
(i \tau-A) c(\tau) x=x, \forall x \in H, \tau \in \mathbb{R} \text {, }
$$

且

$$
c(\tau)(i \tau-A) x=x, \forall x \in D(A),
$$

其中 $D(A)$ 是 $A$ 的定义域. 故 $\{i \tau \mid \tau \in \mathbf{R}\} \subset \rho(A)$, 并且 $R(i \tau ; A)=c(\tau), \forall \tau \in \mathbf{R}$. 此外, 由(4)式有

$$
\|R(i \tau ; A)\| \leqslant\left\|R\left(\sigma_{0}+i \tau ; A\right)\right\| \frac{1}{1-k_{0}} \leqslant \frac{1}{1-k_{\theta}} \sup _{\tau \in \mathbb{R}}\left\|R\left(a_{0}+i \tau ; A\right)\right\|<+\infty,
$$

于是仍由文献 [3]知 $\tau(t)$ 是指数稳定的.

\section{定理 2 的证明 必要性. 显然.}

充分性. 若 $p=1$, 从文献 [3] 可得结论. 设 $p>1$. 反复用 Baire 纲定理可得常数 $M>$ 0 使

$$
\int_{0}^{\infty}|\langle\tau(t) x, y\rangle|^{p} \mathrm{~d} t \leqslant M^{p}\|x\|^{p}\|y\|^{p}, \forall x, y \in H .
$$

设 $\lambda=a+i \tau . \forall \sigma>0$, 由不等式(5)知

$$
\begin{aligned}
\int_{0}^{\infty}\left|e^{-\lambda t}\langle\tau(t) x, y\rangle\right| \mathrm{d} t & \leqslant\left(\int_{0}^{\infty} e^{-q \sigma t} \mathrm{~d} t\right)^{\frac{1}{q}}\left(\int_{0}^{\infty}|\langle\tau(t) x, y\rangle|^{p} \mathrm{~d} t\right)^{\frac{1}{p}} \\
& \leqslant\left(\frac{1}{a q}\right)^{\frac{1}{q}} M\|x\|\|y\|, \forall x, y \in H,
\end{aligned}
$$

其中 $q$ 满足 $\frac{1}{p}+\frac{1}{q}=1$. 由 Riesz 表示定理知存在有界算子 $B(\lambda): H \rightarrow H$, 使

$$
\langle B(\lambda) x, y\rangle=\int_{0}^{\infty} e^{-\lambda t}\langle\tau(t) x, y\rangle \mathrm{d} t, \forall \sigma>0, x, y \in H .
$$

且由不等式(6)有

$$
\|B(\lambda)\| \leqslant M\left(\frac{1}{\sigma q}\right)^{\frac{1}{q}}, \forall \sigma>0 .
$$

令 $\omega_{0}=\lim _{t \rightarrow \infty}(\log \|\tau(t)\|) / t$, 则当 $\sigma>\max \left(\omega_{0}, 0\right)$ 时, 由 $C_{0}$ 半群的 Laplace 反演公式及(7) 式知

$$
B(\lambda)=R(\lambda ; A), \forall \sigma>\max \left(\omega_{0}, 0\right) .
$$

由(7)式及解析函数的延拓法知

$$
\{\lambda \mid \operatorname{Re} \lambda>0\} \subset \rho(A),
$$

且 
从不等式(8)有

$$
B(\lambda)=R(\lambda ; A), \forall \sigma>0 .
$$

$$
0 \leqslant \lim _{a \rightarrow 0^{+}} \sigma \sup _{r \in \mathbb{R}}\|R(a+i \tau ; A)\| \leqslant \lim _{\sigma \rightarrow 0^{+}} M\left(\frac{1}{q}\right)^{\frac{1}{q} \sigma_{p}^{\frac{1}{p}}}=0 .
$$

于是从定理 1 可得所需结论.

定理 3 的证明 充分性. 重复使用 Baire 纲定理可得常数 $M_{1}>0$, 使

$$
\int_{\mathbf{R}}|\langle R(\sigma+i \tau ; A) x, y\rangle|^{p} \mathrm{~d} \tau \leqslant M P\|x\|^{p}\|y\|^{p}, \forall \sigma>0, x, y \in H .
$$

设 $\lambda=a+i \tau$. 显然对任意固定的 $x, y \in H,\langle R(\sigma+i \tau ; A) x, y\rangle$ 是右半平面 $\operatorname{Re} \lambda>0$ 上解 析函数. 任取一固定的 $\lambda$, 使 $\operatorname{Re} \lambda=\sigma>0$. 由解析函数的 Cauchy 公式, 对任意 $0<\rho<$ $\frac{\sigma}{2}$, 有

$$
\begin{aligned}
\langle R(\lambda ; A) x, y\rangle & =\frac{1}{2 \pi i} \oint_{|z-\lambda|=\rho} \frac{\langle R(z ; A) x, y\rangle}{z-\lambda} \mathrm{d} z \\
& =\frac{1}{2 \pi} \int_{0}^{2 \pi}\left\langle R\left(\lambda+\rho e^{i \theta} ; A\right) x, y\right\rangle \mathrm{d} \varphi .
\end{aligned}
$$

(10)式两端同乘 $\rho$,并将 $\rho$ 从 0 到 $\frac{\sigma}{2}$ 积分得

$$
\begin{aligned}
\frac{\sigma^{2}}{8}\langle R(\lambda ; A) x, y\rangle & =\frac{1}{2 \pi} \int_{0}^{\frac{\sigma}{2}} \int_{0}^{2 \pi}\left\langle R\left(\lambda+\rho e^{i \theta} ; A\right) x, y\right\rangle \rho \mathrm{d} \theta \mathrm{d} \rho \\
& =\frac{1}{2 \pi} \iint_{C}\langle R(s+i t ; A) x, y\rangle \mathrm{d} s \mathrm{~d} t,
\end{aligned}
$$

其中积分 域 $C=\left\{(s, t) \mid(s-\sigma)^{2}+(t-\tau)^{2} \leqslant \frac{\sigma^{2}}{4}\right\}$. 由不等式(9)有

$$
\begin{aligned}
\frac{\sigma^{2}}{8}|\langle R(\lambda ; A) x, y\rangle| & \leqslant \frac{1}{2 \pi} \int_{\frac{\sigma}{2}}^{\frac{3 \sigma}{2}} \int_{\tau-\frac{\sigma}{2}}^{\tau+\frac{\sigma}{2}}|\langle R(s+i t ; A) x, y\rangle| \mathrm{d} s \mathrm{~d} t \\
& \leqslant \frac{1}{2 \pi} \int_{\frac{\sigma}{2}}^{\frac{3 \sigma}{2}}\left(\int_{R}|\langle R(s+i t ; A) x, y\rangle|^{p} \mathrm{~d} t\right)^{\frac{1}{p}} \sigma^{\frac{1}{q}} \mathrm{~d} s \\
& \leqslant \frac{M_{1}}{2 \pi} \sigma^{1+\frac{1}{q}}\|x\|\|y\|, \forall \sigma>0, x, y \in H,
\end{aligned}
$$

即

$$
\sigma \|\left(R(\lambda ; A) \| \leqslant \frac{4 M_{1}}{\pi} \sigma^{\frac{1}{q}}, \forall \operatorname{Re} \lambda=\sigma>0 .\right.
$$

于是从定理 1 可得 $\tau(t)$ 的指数稳定性.

必要性. 设 $T(t)$ 是指数稳定的, 则存在 $\eta>0$ 及 $k_{1}>0$, 使

$$
\|T(t)\| \leqslant k_{1} e^{-\eta t}, \forall t \geqslant 0,
$$

从而

$$
\int_{0}^{\infty}|\langle T(t) x, y\rangle|^{2} \mathrm{~d} t \leqslant \frac{k_{1}^{2}}{2 \eta}\|x\|^{2}\|y\|^{2}, \forall x, y \in H_{\bullet}
$$


由 $C_{0}$ 半群的 Lapluce 反演公式有

$$
\langle R(a+i \tau ; A) x, y\rangle=\int_{0}^{\infty} e^{-i s t} e^{-\sigma \tau}\langle T(t) x, y\rangle \mathrm{d} t, \forall \sigma>0, x, y \in H .
$$

令

$$
f(t)= \begin{cases}e^{-\sigma t}\langle T(t) x, y\rangle, & t \geqslant 0, \\ 0, & t<0,\end{cases}
$$

由(12)式和 $f(\cdot) \in L^{2}(\mathrm{R})$, 其中 $L^{2}(\mathrm{R})$ 是由 $\mathbf{R}$ 上平方可积函数所成 Hilbert 空间. 由(13) 式知

$$
\langle R(\sigma+i \tau ; A) x, y\rangle=\sqrt{2 \pi} \hat{f}(\tau), \tau \in \mathbf{R},
$$

其中 $f$ 表示 $f$ 在 $L^{2}(\mathbf{R})$ 中的 Fourier 变换. 由 Fourier 变换是 $L^{2}(\mathbf{R})$ 中线性等距算子知

$$
\langle R(\sigma+i \tau ; A) x, y\rangle \in L^{2}(\mathrm{R}),
$$

即由不等式(12)有

$$
\begin{aligned}
\int_{R}|\langle R(\sigma+i \tau ; A) x, y\rangle|^{2} \mathrm{~d} \tau-2 \pi \int_{R}|\hat{f}(\tau)|^{2} \mathrm{~d} \tau \\
\quad=2 \pi \int_{0}^{\infty}|\langle T(t) x, y\rangle|^{2} \mathrm{~d} t \leqslant \frac{k_{1} \pi}{\eta}\|x\|^{2}\|y\|^{2}, \forall \sigma>0, x, y \in H,
\end{aligned}
$$

即 $p=2$ 使(2)式成立.

\section{参考文献}

[1] Pazy, A., Semigroups of Linear Operators and Applications to Partial Differential Equations, Springer-Verlag, New York, Berlin, Heidelberg, Tokyo, 1983.

[2] Pritchard, A. J., Zabczyk, J., Stability and Stabilizability of Infinize Dimensional Systems, Society for Industrial and Applied Mathematics, 1981, 23(1): 25-50.

[3] Huang Fa-1un (黄发伦), Ann. of Diff. Eqs., 1985, 1(1): 1-55. 